\title{
Hepatic Mucormycosis in a Patient of Acute Lymphoblastic Leukemia: A Case Report with Literature Review
}

\author{
Afaq Ahmad Khan • Vinay Kumaran • \\ Deepali Jain · Fouzia Siraj · Shyam Aggarwal
}

Received: 26 November 2011/Accepted: 1 February 2012/Published online: 14 February 2012

(c) Indian Society of Haematology \& Transfusion Medicine 2012

\begin{abstract}
Mucormycosis is an invasive opportunistic fungal infection associated with a high mortality rate, and normally occurs in immunocompromised patients. It can be encountered during neutropenia following chemotherapy in acute leukemia patients. The common sites involved are rhinocerebral, pulmonary, gastrointestinal and spleen. Hepatic involvement has been reported rarely. We hereby report a case of acute lymphoblastic leukemia who while on chemotherapy developed hepatic mucormycosis and was managed successfully despite its treatment being quite challenging, especially in the context of surgery in a neutropenic sick patient.
\end{abstract}

Keywords Mucormycosis - Acute lymphoblastic leukemia $\cdot$ Amphotericin B

\section{Introduction}

Mucormycosis is a disease caused by order mucorales, a ubiquitous saprophytic mold found in soil and organic matter

\author{
A. A. Khan $(\square)$ \\ Sir Ganga Ram Hospital, R/O 3/17, Old Rajinder Nagar, New \\ Delhi 110060, India \\ e-mail: drafaqak@yahoo.co.in \\ V. Kumaran · D. Jain · F. Siraj · S. Aggarwal \\ Sir Ganga Ram Hospital, Rajinder Nagar, New Delhi 110060, \\ India \\ e-mail: KUMARANVINAY@YAHOO.COM \\ D. Jain \\ e-mail: deepalijain76@gmail.com \\ F. Siraj \\ e-mail: fouziasiraj2009@gmail.com \\ S. Aggarwal \\ e-mail: drshyam_aggarwal@yahoo.com
}

worldwide. The disease in humans is mainly limited to people with risk factors such as neutropenia, immune deficiencies, malignant disease, malnutrition, diabetes, trauma, organ transplantation and iron overload. It rarely affects the liver and it may be difficult to manage these very sick patients.

\section{Case}

A 21 years old boy presented with fever, symptoms related to anemia and bluish spots over the body. His physical examination revealed that he was febrile, had pallor, petechiae, generalized lymphadenopathy and mild hepatosplenomegaly. Complete blood counts showed $\mathrm{Hb}$ of $5.4 \mathrm{~g} /$ $\mathrm{dl}$, platelets of $16000 / \mu \mathrm{l}$ and total leucocyte count of $221,000 / \mu 1$ with $95 \%$ circulating blasts. Bone marrow confirmed acute leukemia with almost complete replacement by myeloperoxidase negative blasts. Flow cytometric immunophenotyping revealed CD10 positive precursor B cell ALL. Besides the supportive treatment, he received induction chemotherapy with following drugs-vincristine, prednisolone, doxorubicin, asparaginase and intrathecal chemotherapy. Post induction he went into remission. He was then started on consolidation with cyclophosphamide, cytarabine and $6 \mathrm{MP}$. Midway during consolidation he developed neutropenic fever (ANC200) for which he required broad spectrum antibiotics including Imepenem, teicoplanin and growth factors (G-CSF). He improved and his consolidation was continued. After the completion of consolidation he was given maintenance treatment with six mercaptopurine and methotrexate. After 2 weeks of maintenance, he complained of failure to thrive and daily evening rise of temperature to $100^{\circ} \mathrm{F}$. His chemotherapy was stopped and he was investigated further. CBC, DLC, renal function tests, chest $\mathrm{X}$-ray were normal as was the 
bone marrow examination. Blood and urine cultures were sterile. Ultrasound abdomen revealed space occupying lesions in liver. Liver enzymes were slightly raised. Contrast enhanced CT chest was within normal limits but $\mathrm{CT}$ abdomen confirmed space occupying lesions in liver. These lesions were multiple, coalescent, hypodense with enhancing walls predominantly involving segment 5 and 8 of the right lobe (Fig. 1).The hepatic vasculature and biliary radicals were unremarkable. Serum galactomannan assay was negative. Fine needle aspiration of those lesions revealed multiple branched hyphae, suggestive of mucormycosis. Patient's chemotherapy was withheld and he was started on amphotericin B deoxycholate $50 \mathrm{mg} /$ day. Patient's condition deteriorated. A surgical consultation was made. They advised surgical resection of the involved part, for which family members were initially reluctant, but later on gave the high risk consent for surgery. Patient underwent surgery. The involved portion was removed and sent for histopathological examination. Gross examination revealed multiple greyish brown necrotic nodules (Fig. 2). Microscopy showed necrotizing acute inflammatory infiltrate with presence of multiple, broad, sparsely septate fungal hyphae with right angle branching highly suggestive of hepatic mucormycosis (Fig. 3). After the surgery patient received 3 grams of amphotericin B as a total cumulative dose over a period of 2 months. He had full recovery with repeat CT abdomen showing normal liver parenchyma. Because of the cessation of chemotherapy for about 2 months he started with blasts again in the peripheral blood. He then received chemotherapy with FLAG-IDA and achieved complete remission again.

\section{Discussion}

Mucormycosis is a fungal infection representing $12 \%$ of all documented filamentous mycosis. Portals of entry for mucorales include sinuses, lungs, gastrointestinal tract and skin.

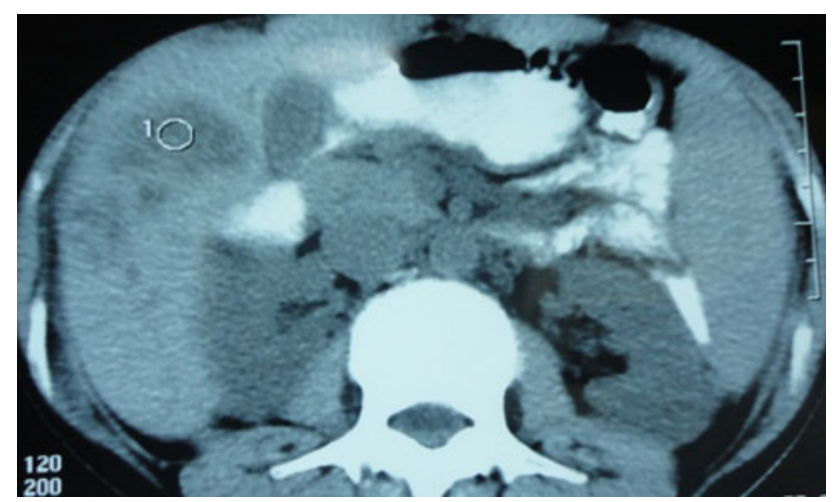

Fig. 1 CT scan abdomen showing multiple small coalescent hypodense lesions with enhancing walls in the right hepatic lobe, predominantly involving segment 5 and 8 parenchyma

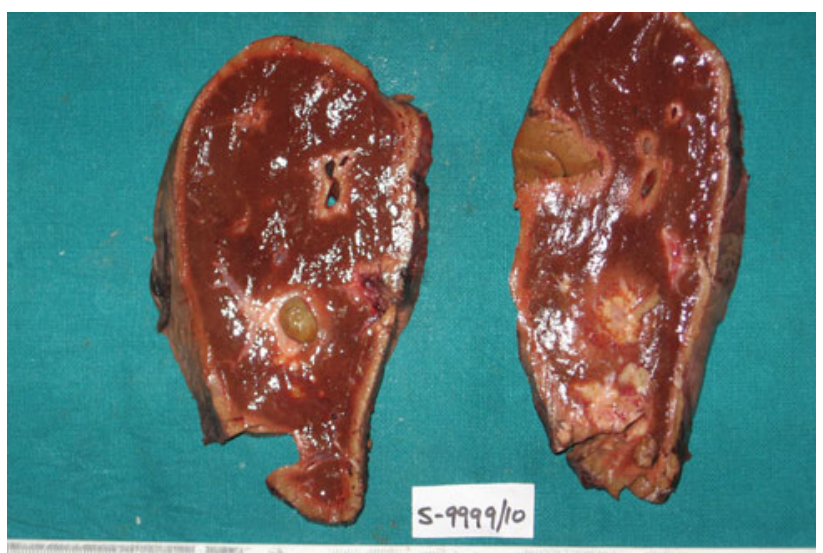

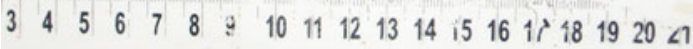

Fig. 2 Cut surface of resected portion of liver showing multiple greyish brown nodules with extensive necrosis

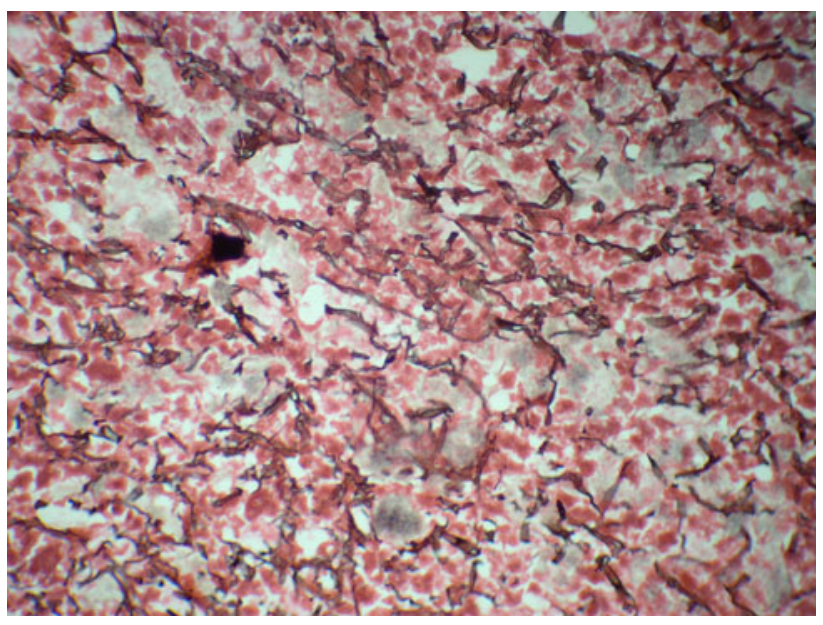

Fig. 3 Photomicrograph showing presence of numerous broad, sparsely septate fungal hyphae with right angle branching; highly suggestive of hepatic mucormycosis (Silver methenamine stain, $\times 20$ )

Hepatic involvement is commonly presented with pulmonary or gastrointestinal infection and is considered a part of the disseminated disease [1]. Disseminated mucormycosis is the most severe form and is said to be uniformly fatal [2]. The time lost before a correct diagnosis is made may be critical for survival.

In acute leukemia, clinical presentation of hepatic mucormycosis is predominantly that of a persistent neutropenic fever, despite being on broad spectrum antibiotics. Patients are sicker, may have jaundice, abdominal pain and right hypochondriac tenderness. On evaluation, may show hyperbilirubinemia, transaminitis or high alkaline phosphatase. CT scan often shows hypodense mass lesion. The hypodense hepatic lesion surrounding vessels without a mass effect suggest an angioinvasive organism causing fungal thrombosis and involvement of perivascular area subsequently. The CT findings are not pathognomonic but are valuable in narrowing the differential diagnosis [2]. 
Culture and pathologic examinations of biopsy specimen provides the only definite diagnoses of mucormycosis. Mucorales appears in tissue as irregularly shaped, broad nonseptate hyphae with the right angle branching. The most characteristic features are perivascular and blood vessel invasions that result in arterial thrombosis and subsequent necrosis [1].

The standard therapy for invasive mucormycosis is a combined medical-surgical approach [3, 4]. High doses of amphotericin B should be used, rapidly reaching $1.0 \mathrm{mg} / \mathrm{kg}$ daily. Prolonged courses ( $>6$ week) of amphotericin B are recommended, with total doses ranging between 1.5 and $3.0 \mathrm{~g}$, according to the patient's underlying conditions[5].

Patient may need extensive surgical debridement for the successful treatment of this condition. With carefully coordinated surgical and medical treatment it should be possible to reduce the high mortality associated with this condition. Suh et al. reported a case of hepatic and intestinal mucormycosis in a patient of acute lymphoblastic leukemia who eventually died despite surgery and antifungal therapy [1]. Lee et al. reported a similar case of ALL with intestinal and hepatic mucormycosis who succumbed to progressive liver failure despite undergoing intestinal surgery and receiving amphotericin B [6]. Roden et al. reviewed 929 cases of zygomycosis and found that mortality varied with the site of infection: $96 \%$ of patients with disseminated disease died, $85 \%$ with gastrointestinal infection died, and $76 \%$ with pulmonary infection died. Survival was $3 \%$ (eight of 241 patients) for cases that were not treated, $61 \%$ (324 of 532) for cases treated with amphotericin B deoxycholate, 57\% (51 of 90) for cases treated with surgery alone, and 70\% (328 of 470) for cases treated with antifungal therapy and surgery [7]. In contrast to high mortality shown by previous case reports, Li et al. reported a case of hepatic and adrenal mucormycosis in an immunocompetent patient who was successfully treated with amphotericin B and surgery [8]. The successful treatment in our case emphasizes the need to have high index of suspicion, need for early diagnosis and combined medical and surgical approach.

\section{References}

1. Suh IW, Park CS, Lee MS et al (2000) Hepatic and small bowel mucormycosis after chemotherapy in a patient with acute lymphocytic leukemia. J Korean Med Sci 15:351-354

2. Hagspiel KD, Kempf W, Hailemariam S, Marincek B (1995) Mucormycosis of the liver: CT findings. AJR Am J Roentgenol 165:340-342

3. Pagano L, Ricci P, Tonso A et al (1997) Mucormycosis in patients with haematological malignancies: a retrospective clinical study of 37 cases. GIMEMA infection program (Gruppo Italiano Malattie Ematologiche Maligne dell'Adulto). Br J Haematol 99:331-336

4. Jiménez C, Lumbreras C, Aguado JM et al (2002) Successful treatment of mucor infection after liver or pancreas-kidney transplantation. Transplantation 73:476-480

5. Marcó del Pont J, De Cicco L, Gallo G, Llera J, De Santibanez E, D'agostino D (2000) Hepatic arterial thrombosis due to mucor species in a child following orthotopic liver transplantation. Transpl Infect Dis 2:33-35

6. Lee JH, Ha HK, Yoo E, Yang SK, Min YI, Auh YH (2000) CT and sonographically guided biopsy in a patient with intestinal mucormycosis. AJR Am J Roentgenol 175(1):129-131

7. Roden MM, Zaoutis TE, Buchanan WL et al (2005) Epidemiology and outcome of zygomycosis: a review of 929 reported cases. Clin Infect Dis 41:634-653

8. Li KW, Wen TF, Li GD (2010) Hepatic mucormycosis mimicking hilar cholangiocarcinoma: a case report and literature review. World J Gastroenterol 16:1039-1042 